\title{
Intrinsic and external determinants of antibiotic prescribing: a multi-level path analysis of primary care prescriptions in Hubei, China
}

\author{
Chenxi Liu', Chaojie Liu², Dan Wang ${ }^{1}$ and Xinping Zhang ${ }^{1 *}$ (i)
}

\begin{abstract}
Background: Irrational use of antibiotics is a major driver of development of antibiotic resistance, which heavily threatens population health worldwide. Understanding the mechanism of physician's antibiotic prescribing decisions is increasingly highlighted to promote prudent use of antibiotics. Therefore, the current study aimed to fill the gap, modelling physician's antibiotic prescribing and identifying the potential intrinsic and external determinants of antibiotic prescribing in primary care.

Methods: A total of 428,475 prescriptions from 499 prescribers in 65 primary care facilities in Hubei of China were audited. Teixeira Antibiotic Prescribing Behavioral Model (TAPBM) was referred as theoretical basis to identify intrinsic and external predictors of antibiotic prescriptions. A questionnaire survey was conducted, covering potential physician's intrinsic determinants (knowledge, attitudes and individual characteristics) and external factors both in individual level (patient pressure, time pressure and financial incentives) and institutional level (setting and local socio-economic development). A two-level path analysis was performed linking potential determinants of antibiotic use with physician's actual practices.
\end{abstract}

Results: About $44.28 \%$ of the prescriptions contained antibiotics, with $9.28 \%$ containing two or more antibiotics. The multi-level path analysis revealed that knowledge was a significant predictor of attitudes $(\beta=0.154, p<0.05)$, but higher knowledge and attitudes failed to translate into antibiotic prescribing practices ( $\beta=-0.076-0.039, p>0.05)$. Instead, external factors played a more important role and physician's antibiotic use was significantly associated with patient pressure $(\beta=0.102, p=0.022)$, time pressure $(\beta=-0.164, p=0.002)$, financial incentives $(\beta=-0.133--0.155$, $p=0.027$ ) and institutional environments (rural area, $\beta=0.408, p=0.002$; and high socioeconomic setting, $\beta=-0.641$ $-0.578, p<0.001)$. The prescribers who were male $(\beta=-0.168, p=0.007)$ or had lower qualification $(\beta=-0.114, p=$ 0.028 ) were also more likely to prescribe antibiotics than others.

Conclusion: Antibiotic prescribing practices are complex process and associated with both intrinsic (prescriber) and external (patients and institutional environment) factors. A systematic approach is required to curb over-prescription of antibiotics. Apart from educating prescribers, it is equally important, if not more, to educate patients, break incentives and nurture professional culture within organization to reduce the overuse of antibiotics.

Keywords: Primacy care, Antibiotic prescribing, Path analysis, Knowledge-attitudes-practices, China

\footnotetext{
* Correspondence: xpzhang602@hust.edu.cn; xpzhang602@163.com

${ }^{1}$ School of Medicine and Health Management, Tongji Medical School,

Huazhong University of Science and Technology, Wuhan, Hubei, China

Full list of author information is available at the end of the article
}

(c) The Author(s). 2019 Open Access This article is distributed under the terms of the Creative Commons Attribution 4.0 International License (http://creativecommons.org/licenses/by/4.0/), which permits unrestricted use, distribution, and reproduction in any medium, provided you give appropriate credit to the original author(s) and the source, provide a link to the Creative Commons license, and indicate if changes were made. The Creative Commons Public Domain Dedication waiver (http://creativecommons.org/publicdomain/zero/1.0/) applies to the data made available in this article, unless otherwise stated. 


\section{Backgrounds}

Antibiotic resistance (AR), one of the most serious public health issues, threatens population health and socioeconomic development of all nations over the world [1]. It not only jeopardizes our ability to prevent and treat microbial infections, but also put many common medical procedures (e.g. caesarean sections) potentially fatal again, for which antibiotics are commonly used to prevent potential infections. It was projected that $A R$ will contribute to 10 million deaths in 2050 if no effective actions are taken, surpassing cancer and becoming the leading cause of death [2].

Irrational use of antibiotics is a major driver fueling the development of AR [3, 4]. Promoting prudent antibiotic use is considered as a core measure to address this global threat [1]. For patients, antibiotics are commonly prescription-required medicines. Therefore, rational prescribing from physicians plays a fundamental role for prudent use of antibiotics [5]. Unfortunately, inappropriate and over prescriptions of antibiotics are prevalent worldwide. For example, 30\% of antibiotic prescriptions in outpatient care in the US were deemed unnecessary [6]. In China, this figure was estimated to be as high as $60 \%$ [7].

Many studies have attempted to understand how and why physicians prescribe antibiotics irrationally $[8,9]$. Knowledge and attitudes are perhaps the most frequently explored predictors of antibiotic prescribing [10-24]. These studies often suffered from some common methodological limitations. For example, they examined the theoretical knowledge of physicians and their intended use of antibiotics for simulated cases $[9,21]$. However, prescribing practices are subject to the influence of a much broader range of factors. Our understanding on actual antibiotic prescribing practices is still limited $[8,9,25]$.

Teixeira suggested a comprehensive framework for studying prescribing practices [8]. It considers the influence of both intrinsic factors (e.g. knowledge and attitudes of prescribers) and external factors (e.g. requests from patients, employers and governments) on prescribing practices. The Teixeira Antibiotic Prescribing Behavioral Model (TAPBM) also tries to explain the complicated connections among multiple factors, which may involve precedence or compromise.

Although the TAPBM has been widely endorsed by the research community [8], there is a lack of empirical studies testing the model, possibly due to difficulties to link different sources of data. This study aimed to test the TAPBM through linking the survey data of prescribers to their prescriptions. The findings will contribute to the existing debate on determinants of antibiotic prescribing practices, filling the gap in the literature.

\section{Methods}

\section{Study setting}

This study was conducted in Hubei, a province in central China covering a land of 185.9 thousand $\mathrm{km}^{2}$ and 59.02 million populations. Hubei had a gross domestic product (GDP) of $\$ 8915$ per capita in 2017, ranking at number 11 among all (34) provinces in China. It is classified as a middle-high income region based on the criteria of the World Bank [26].

The study setting was restricted to primary care facilities, which included both urban community health centers (UCHCs) and rural township health centers (RTHCs). In 2017, Hubei had 347 UCHCs and 1137 RTHCs, receiving 23.03 million and 56.00 million outpatient visits, respectively [26]. Over-prescription of antibiotics was prevalent in these facilities: $65 \%$ of prescriptions contained antibiotics and $20 \%$ involved two or more antibiotics [27].

\section{Theoretical framework}

This study adopted the TAPBM as an overarching framework. The TAPBM extended the widely-accepted Knowledge-Attitudes-Practices (KAP) theory and included considerations of external determinants of antibiotic prescribing practices [8].

Intrinsic determinants: knowledge and attitudes are the core of intrinsic determinants of antibiotic prescribing practices. Knowledge can influence prescribing behaviors directly or indirectly through influencing attitudes [8]. The knowledge and attitudes of prescribers can be shaped by many individual characteristics $[8,9]$, such as gender, qualifications, clinical expertise, continuing education, and years of practices.

External determinants: when physicians prescribe medicines, they are likely to take considerations of many external factors apart from relevant clinical standards and guidelines. Pressures from patients, peers, employers, governments, and the public may sway antibiotic prescribing decisions. These factors can exert impacts on prescribing practices directly or indirectly through influencing attitudes [8].

Previous studies showed that patient requests for antibiotics increased antibiotic prescribing dramatically, especially when the physicians felt that they did not have enough time to explain details to their patients $[28,29]$. It has been well documented that financial incentives can also drive antibiotic prescribing in primary care in China. Such perverse incentives are particularly strong for the physicians with a lower household income [30].

Formal and informal institutional and system arrangements also play an important role in shaping prescribing decisions. Several studies in the European 
countries showed that antibiotic prescribing practices are associated with the culture of an organization (shared values and behaviors among employees) [31, 32] and the culture of a nation (e.g. tolerance of uncertainty and status distinction) [33]. Empirical evidence in China showed that over-prescription of antibiotics is more prevalent in primary care facilities in rural [7] and socioeconomically disadvantaged regions [34, 35] compared with those in urban and better-off regions.

In this study, the TAPBM framework was adapted considering the availability of relevant data and measurements. We adopted a two-level modelling approach, covering the effects of individual (physician) factors and institutional (cluster-level) factors. The individual-level measurements included both intrinsic (e.g. characteristics of prescribers) and external (e.g. perceived pressures) indicators. The institutional-level measurements were represented by two proxy indicators: urban/rural location and socioeconomic status of the servicing community (Fig. 1). We chose a two-level model for two reasons. First, a cluster sampling strategy was adopted to select study participants. Second, cluster effects were significant as measured by the intra-cluster correlation coefficient (ICC): 0.004 for knowledge, 0.094 for attitudes, and 0.395-0.408 for antibiotic prescribing practices. Institutional variations in individual knowledge, attitudes and prescribing practices were deemed random (depicted using a black dot in Fig. 1), and their random intercepts were predicted by the two institutional indicators.

\section{Measurements}

\section{Outcome measures}

Two indicators were calculated to measure antibiotic prescribing practices in line with the World Health Organization (WHO) recommendations [36]:

- Percentage of prescriptions containing antibiotics $=$ Number of prescriptions containing antibiotics / Total prescriptions $\times 100 \%$;

- Percentage of prescriptions with combined use of antibiotics $=$ Number of prescriptions containing two or more antibiotics / Total prescriptions $\times 100 \%$;

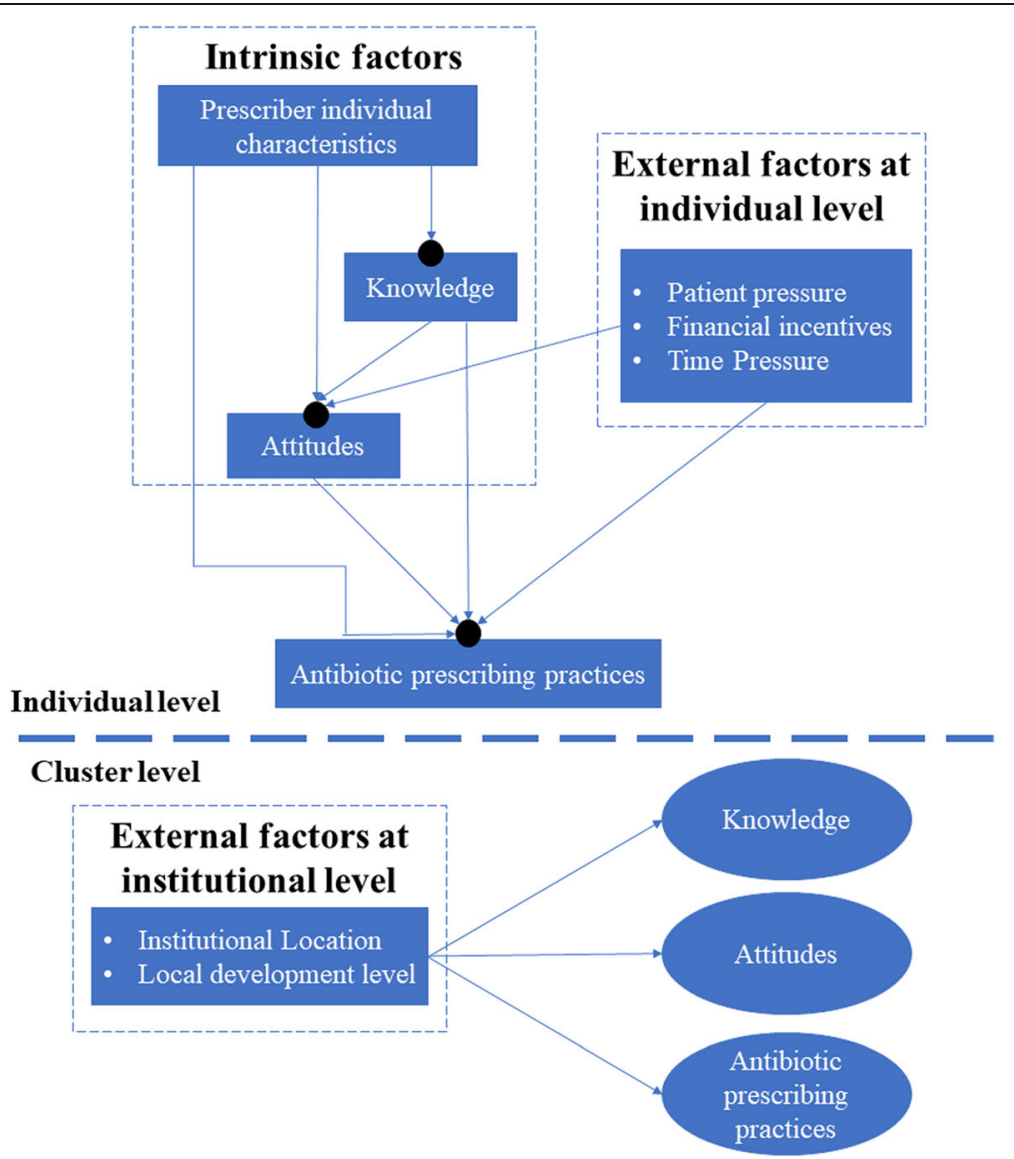

Fig. 1 Antibiotic prescribing behavioral model adapted from the TAPBM. Institutional variations in individual knowledge, attitudes and prescribing practices were treated as a random effect and depicted using black dots. Their random intercepts were predicted by two institutional indicators 


\section{Predictors of outcome measures}

Individual-level intrinsic factors Knowledge: Antibiotic prescribing knowledge was measured with 11 items that were commonly used in previous studies [13, 22, 37], asking the physician respondents to make a prescribing decision under various scenarios, such as for patients with non-febrile diarrhea, upper respiratory tract infections, methicillin resistant staphylococcus, and bacterial pneumonia. Each scenario was provided with 4 or 5 prescribing options, with only one correct answer. An "unknown" option was also listed to discourage guessing. A summed score of knowledge about antibiotic prescribing was calculated, ranging from 0 to 11 . A higher score indicates better knowledge.

Attitudes: Attitudes of the physician respondents were measured using the 11-item Knowledge and Attitudes regarding Antibiotics and Resistance (KAAR-11) scale, which has been fully validated in previous studies [38]. The KAAR-11 asked respondents to rate their opinions on a four-point Likert scale (ranging from 0 "totally agree" to 4 "totally disagree") about the excuses for irrational prescriptions of antibiotics, such as satisfying patient requests, avoiding patient complaints, ignoring or blaming others for AR. A summed score of KAAR-11 was calculated, ranging from 0 to 44 . A higher score indicates a more favorable attitude to rational prescribing of antibiotics [38].

Individual characteristics of prescribers: These included the demographic characteristics (gender and education) and the professional experiences (years of clinical practices, professional title, and training on antibiotic prescriptions) of the participants. All of the physician respondents were general practitioners. Therefore, clinical expertise was excluded in the analysis.

Individual-level external factors Perceived patient pressure: Respondents were asked "how many percent of patients you see expect antibiotic prescriptions" and "how often the patient expectation influences your decision of antibiotic prescribing". The latter was rated on a five-point Likert scale ranging from " $1=$ always" to " $0=$ never". Scores of the two questions were multiplied as an indication of patient pressure (ranging from 0 to 100).

Time pressure: Respondents were asked to estimate the average duration (minutes) of consultations for each patient visit, with a lower duration indicating higher time pressure.

Household income: Respondents were asked to estimate their annual household income (Chinese Yuan $¥$ ) over the previous year. The household income was classified into four categories: <40,000; 40,000-79,999; 80, 000-119,999; $\geq 120,000$.
Institutional-level measures The characteristics of the participating primary care institutions were measured with two indicators: rural/urban location and socioeconomic status of the servicing community. All of the participating primary care facilities were public institutions governed by the same policy and regulatory framework. The socioeconomic status was classified based on GDP per capita of their local districts: high (top four districts) vs low.

\section{Pilot survey}

The questionnaire was pilot tested in 23 physicians from 3 primary care facilities. They were asked to explain why they chose or did not choose certain answers. The feedback was used to modify the questionnaire. Of the 30 tested questionnaire items, 9 were rephrased and no items were deleted or added (the final instrument could be found in the Additional file 1: Table S1).

\section{Sampling and data collection}

A stratified cluster sampling strategy was adopted to select study participants. A total of 65 primary care facilities were randomly selected from 3 urban and 6 rural districts corresponding to the distribution of primary care facilities in Hubei. The participating primary care institutions represented 5.5\% (19/347) of UCHCs and $4.1 \%(46 / 1137)$ of RTHCs, respectively. Details about the sampling approach have been published elsewhere [39].

The physicians who prescribed antibiotics independently in the participating primary care facilities were eligible for this study. To minimize bias, only those who prescribed $\geq 100$ prescriptions three months prior to the commencement of the questionnaire survey of prescribers were invited to participate in this study.

Extraction of prescription data Prescription data from January 1st to March 31st, 2018 were extracted either from the participating primary care institutions or from the medical administration system of the local governments. These included the name of the institution, the ID number of the prescriber, patient ID, date of the prescription, and the type, volume and price of the medicines prescribed.

Questionnaire survey A questionnaire survey of prescribers in the participating primary care institutions was conducted from April 23rd to June 6th, 2018. A pair of trained investigators visited each primary care facility and invited all of the physicians working at the time to complete the questionnaire. An informed written consent was obtained before the respondents self-completed the questionnaire. The survey took about $15 \mathrm{~min}$. Completed questionnaires were returned to the investigators immediately. The investigators examined the completeness of the returned questionnaires. Missing items, if 
existed, were re-filled through a supplementary interview. A token gift (\$1.65) was given to the participants.

In total, 712 physicians agreed to participate in the survey, representing $93.44 \%$ of all physicians in the participating institutions. About 664 complete questionnaires were returned, of which 499 met the inclusion criteria and passed logic check. This resulted in an effective response rate of $70.08 \%$.

Data mapping The prescription outcome indicators were calculated from the extracted prescription database and then mapped into the questionnaire survey data in line with the ID number of the prescribers. The two institutional variables were obtained from the classification system of the statistics bureau of Hubei government and mapped into the questionnaire data in line with the name of the institutions.

\section{Data analysis}

We compared the differences of respondents between the urban and rural settings, using Chi-square (or Fisher's exact) tests for binary variables, Wilcoxon rank-sum tests for continuous variable without a normal distribution, and Student $t$ tests for continuous variables with a normal distribution.

A two-level path analysis was performed to test the TAPBM using a mixed effect model. Institutional variations in the individual knowledge, attitudes and prescribing practices were treated as a random effect, with the random intercepts being further predicted by the institutional (cluster-level) variables. The analysis adopted the maximum likelihood with robust standard error (MLR) estimation, which can be applied for variables without a normal distribution. The fitness of data into the model was assessed using three criteria [40, 41]: $\operatorname{RMSEA}<0.08$, $\mathrm{CFI}>0.95$, and $\mathrm{SRMR}<0.08$.

The statistical analyses were performed using STATA (version 12.0) and Mplus (version 6.0). A $p$ value $<0.05$ was considered statistically significant.

\section{Results}

\section{Characteristics of survey respondents}

About $21.64 \%$ of respondents worked in the UCHCs. Over half of the UCHC respondents were women compared with only $23.53 \%$ in the RTHCs $(p<0.001)$. Compared with the UCHC respondents, those from the RTHCs were younger, had lower qualifications, lower professional titles, shorter experiences in clinical practices, and lower household income (Table 1). Over three quarters $(78.76 \%)$ of the RTCH respondents attended training programs on antibiotic prescribing in 2017, compared with $62.96 \%$ of respondents from the UCHCs $(p=0.001)$.

\section{Antibiotic prescribing practices}

The study participants prescribed 428,475 prescriptions over the three-month period: $44.28 \%(189,719)$ contained antibiotics and $9.28 \%(39,778)$ contained two or more antibiotics. Those from the RTHCs were more likely to prescribe antibiotics than their UCHC counterparts. But the differences were only statistically significant in the combined use of antibiotics $(p<0.001)$ (Table 2).

The respondents had a mean knowledge score of 6.11 $(\mathrm{SD}=1.46)$ about rational antibiotic prescriptions. The percentage of respondents giving a correct prescribing answer was very low: $5.01 \%$ for upper respiratory tract infections, $11.22 \%$ for renal failure, $29.06 \%$ for methicillin resistant staphylococcus, $46.49 \%$ for bacterial pneumonia, and $41.28 \%$ for antibiotic treatments crossing the blood-brain barrier. No overall knowledge difference appeared between the UCHC and RTHC respondents $(p=0.073)$, although those from the UCHCs were more likely to give a correct answer to antibiotic prescriptions for anaerobes $(p=0.048)$ and to the standards of antibiotic use in primary care $(p=0.003)$.

The respondents had a mean KAAR-11 score of 27.56 $(\mathrm{SD}=3.46)$. Compared with the UCHC respondents, those from the RTHCs were more likely to recognize AR as a public health problem $(p=0.006)$, but blame others for the development of AR $(p=0.017)$, and prescribe antibiotics for defensive purpose $(p<0.05)$.

The study participants perceived high levels of patient pressure on antibiotic prescribing. On average, over half (54.91\%) of the patients were reported to have an expectation on antibiotic prescriptions. This resulted in a mean patient pressure score of $25.63 \%(S D=21.41 \%)$ weighted by perceived influence of patient requests. The RTHC physicians perceived higher patient expectations $(p=0.001)$ and greater impacts of patient pressure $(p=0.026)$ compared with their UCHC counterparts.

On average, the physician respondents spent $10.58 \mathrm{~min}$ $(\mathrm{SD}=6.47)$ for each patient consultation. The length of patient consultation was longer in the UCHCs than in the RTHCs $(p=0.007)$, indicating a lower time pressure.

\section{Determinants of antibiotic prescribing behaviors}

A good fitness of the data into the TAPBM model (Fig. 2) was evident: $\mathrm{RMSEA}=0.037(<0.08), \mathrm{CFI}=0.994(>0.95)$, SRMR $<0.08$ at both individual and cluster levels.

Intrinsic factors: individual knowledge and attitudes did not predict antibiotic prescribing practices $(\beta$ : $-0.076-$ $0.039, p>0.05$ ), although better knowledge was associated with positive attitudes toward rational use of antibiotics ( $\beta=0.154, p=0.001)$. Female physicians were less likely to prescribe antibiotics $(\beta=-0.168, p=0.007)$ but more likely to use two or more antibiotics $(\beta=0.194, p<0.001)$ 
Table 1 Characteristics of study participants

\begin{tabular}{|c|c|c|c|c|}
\hline Characteristics & Overall & Urban Community Health Center & Rural Township Health Center & $p^{*}$ \\
\hline Number of respondents & 499 & 108 & 391 & - \\
\hline \multicolumn{4}{|l|}{ Gender, n (\%) } & $<0.001$ \\
\hline Men & $352(70.54)$ & $53(49.07)$ & $299(76.47)$ & \\
\hline Women & $147(29.46)$ & $55(50.93)$ & $92(23.53)$ & \\
\hline Age, Years (Mean $\pm S D)$ & $43.38 \pm 9.59$ & $48.37 \pm 10.26$ & $42.00 \pm 8.93$ & $<0.001$ \\
\hline Years of clinical practices & $16.28 \pm 10.10$ & $18.69 \pm 11.40$ & $15.61 \pm 9.62$ & 0.015 \\
\hline \multicolumn{4}{|l|}{ Qualification } & $<0.001$ \\
\hline No degree & $42(8.42)$ & $8(7.41)$ & $34(8.70)$ & \\
\hline Associate degree & $266(53.31)$ & $38(35.19)$ & $228(58.31)$ & \\
\hline University degree & $191(38.28)$ & $62(57.41)$ & 129 (32.99) & \\
\hline \multicolumn{4}{|l|}{ Professional title } & $<0.001$ \\
\hline Junior & $257(51.50)$ & $28(25.93)$ & $229(58.57)$ & \\
\hline Middle & $191(38.28)$ & $53(49.07)$ & $138(35.29)$ & \\
\hline Senior & $51(10.22)$ & $27(25.00)$ & $24(6.14)$ & \\
\hline \multicolumn{4}{|c|}{ Annual household income $\left(¥^{+}\right)$} & $<0.001$ \\
\hline$<40,000$ & $143(28.66)$ & $17(15.74)$ & $126(32.23)$ & \\
\hline $40,000-79,999$ & $253(50.70)$ & $47(43.52)$ & $206(52.69)$ & \\
\hline $80,000-119,999$ & $77(15.43)$ & $29(26.85)$ & $48(12.28)$ & \\
\hline$\geq 120,000$ & $26(5.21)$ & $15(13.89)$ & $11(2.81)$ & \\
\hline \multicolumn{4}{|c|}{ Training on antibiotic prescribing in 2017} & 0.001 \\
\hline Attended & $374(74.95)$ & $68(62.96)$ & $306(78.26)$ & \\
\hline Not attended/Not awar & $125(25.05)$ & $40(37.04)$ & $85(21.74)$ & \\
\hline
\end{tabular}

"Wilcoxon-Mann-Whitney tests (continuous and ordinal variables) or Chi-square tests (binary variables); ${ }^{+} ¥$ represented the Chinese unit of currency, Renminbi (RMB)

compared with their male counterparts. Higher qualifications were associated with less prescriptions of antibiotics $(\beta=-0.114, p=0.028)$. Professional title, experience of clinical practices, and continuing education were not significant predictors $(p>0.05)$ on knowledge, attitudes and practices in antibiotic prescribing (omitted in Fig. 2).

External factors: perceived higher patient pressure was associated with negative attitudes towards rational use of antibiotics $(\beta=-0.165, p<0.001)$ and higher use of antibiotics $(\beta=0.102, p=0.022)$. Higher time pressure was associated with higher use of antibiotics $(\beta=$ $-0.164, p=0.002)$, although it failed to predict attitudes toward rational use of antibiotic $(\beta=-0.091, p>0.05)$. Higher household income was associated with lower use of antibiotics $(\beta=-0.133, p=0.027)$ and lower combined use of antibiotics $(\beta=-0.155, p=0.012)$.

At the cluster-level, rural location was a significant predictor of higher use of antibiotics $(\beta=0.408, p=0.002)$. The facilities servicing communities with a higher socioeconomic status were less likely to prescribe antibiotics $(\beta=-0.641, p<0.001)$ and less likely to use combined antibiotics $(\beta=-0.578, p<0.001)$. The institutional variables did not predict the knowledge and attitudes of physicians $(p>0.05)$.

\section{Discussions \\ Main findings}

This study confirmed that antibiotic prescribing practices involve complex mechanisms and the TAPBM can be used for exploring determinants of antibiotic prescriptions, including both intrinsic and external factors at the individual and institutional levels.

High levels of antibiotic prescriptions are evident in primary care in Hubei: $44.28 \%$ involved at least one antibiotic. Although this represents a decline of antibiotic prescriptions in general [27], it is still much higher than what has been recommended by the Chinese government (20\%) and the World Health Organization (30\%) [42]. In this study, the physicians demonstrated poor knowledge about antibiotic prescribing. Although poor knowledge does not predict antibiotic prescribing directly, it may leave greater opportunities for external factors to sway prescribing decisions. We found that time pressure and patient requests are associated with higher levels of antibiotic prescriptions. Antibiotic prescribing practices are 
Table 2 Antibiotic prescriptions and associated factors in primary care

\begin{tabular}{|c|c|c|c|c|}
\hline & Overall & UCHCS & RTHs & $p^{*}$ \\
\hline \multicolumn{5}{|l|}{ Antibiotic prescribing practices of individual physicians (Mean \pm SD) } \\
\hline Percentage of prescriptions containing antibiotics (\%) & $41.45 \pm 20.13$ & $39.55 \pm 23.35$ & $41.97 \pm 19.15$ & 0.066 \\
\hline Percentage of prescriptions containing two or more antibiotics (\%) & $10.23 \pm 10.53$ & $7.00 \pm 9.82$ & $11.12 \pm 10.56$ & $<0.001$ \\
\hline \multicolumn{5}{|l|}{ Number (\%) of correct answers to antibiotic prescribing knowledge } \\
\hline K1: Non-febrile diarrhea & $475(95.19)$ & $102(94.44)$ & $373(95.40)$ & 0.682 \\
\hline K2: Upper respiratory tract infections & $25(5.01)$ & $3(2.78)$ & $22(5.63)$ & 0.320 \\
\hline K3: Renal failure & $56(11.22)$ & $8(7.41)$ & $48(12.28)$ & 0.156 \\
\hline K4: Pregnant patients & $482(96.59)$ & $104(96.30)$ & $378(96.68)$ & 0.770 \\
\hline K5: Anaerobes & $485(97.19)$ & $108(100.00)$ & $377(96.42)$ & 0.048 \\
\hline K6: Methicillin resistant staphylococcus & $145(29.06)$ & $35(32.41)$ & $110(28.13)$ & 0.386 \\
\hline K7: Crossing the blood-brain barrier & $206(41.28)$ & $50(46.30)$ & $156(39.90)$ & 0.232 \\
\hline K8: Bacterial pneumonia & $232(46.49)$ & $56(51.85)$ & $176(45.01)$ & 0.207 \\
\hline K9: Reducing complications of upper respiratory tract infections & $263(52.71)$ & $56(51.85)$ & $207(52.94)$ & 0.841 \\
\hline K10: Administration of Aminoglycosides & $305(61.12)$ & $69(63.89)$ & $236(60.36)$ & 0.505 \\
\hline K11: Standards of antibiotic use in primary cares & $375(75.15)$ & $93(86.11)$ & $282(72.12)$ & 0.003 \\
\hline Summed knowledge score (Mean \pm SD) & $6.11 \pm 1.46$ & $6.33 \pm 1.42$ & $6.04 \pm 1.46$ & 0.073 \\
\hline \multicolumn{5}{|l|}{ Score of attitudes toward rational antibiotic prescribing (Mean \pm SD) } \\
\hline A1: Antibiotic resistance is a major public health problem in my setting & $3.02 \pm 0.90$ & $2.79 \pm 1.01$ & $3.09 \pm 0.86$ & 0.006 \\
\hline A2: It is useful to wait for a microbiology result when treating infections & $3.34 \pm 0.60$ & $3.34 \pm 0.66$ & $3.34 \pm 0.59$ & 0.710 \\
\hline A3: One antibiotic prescription does not influence the development of AR & $3.04 \pm 0.82$ & $2.98 \pm 0.96$ & $3.06 \pm 0.78$ & 0.781 \\
\hline A4: New antibiotics will be created to solve AR problems & $1.54 \pm 0.94$ & $1.74 \pm 1.07$ & $1.48 \pm 0.89$ & 0.017 \\
\hline A5: The use of antibiotics in animals is a major cause of AR & $2.60 \pm 0.91$ & $2.71 \pm 0.81$ & $2.57 \pm 0.94$ & 0.219 \\
\hline A6: Broad-spectrum antibiotics are preferred for infections in doubt & $1.99 \pm 1.02$ & $2.22 \pm 1.02$ & $1.92 \pm 1.01$ & 0.007 \\
\hline A7: Antibiotics are often prescribed for patients untrackable & $2.89 \pm 0.96$ & $3.05 \pm 0.95$ & $2.85 \pm 0.96$ & 0.035 \\
\hline A8: It is best to prescribe antibiotics if bacterial infections are uncertain & $2.87 \pm 0.83$ & $3.01 \pm 0.80$ & $2.83 \pm 0.84$ & 0.042 \\
\hline A9: Antibiotics are often prescribed due to patient demands & $2.86 \pm 0.91$ & $2.77 \pm 0.88$ & $2.88 \pm 0.92$ & 0.161 \\
\hline A10: Patients will get antibiotics from a pharmacy even without my prescriptions & $1.19 \pm 0.94$ & $1.19 \pm 0.96$ & $1.20 \pm 0.93$ & 0.938 \\
\hline A11: Amoxicillin is effective for most respiratory infections in primary care & $2.22 \pm 1.01$ & $2.25 \pm 1.05$ & $2.21 \pm 1.00$ & 0.737 \\
\hline Summed attitudes score & $27.56 \pm 3.46$ & $28.05 \pm 3.52$ & $27.42 \pm 3.43$ & 0.097 \\
\hline \multicolumn{5}{|l|}{ Perceived patient pressure (Mean \pm SD) } \\
\hline Percentage of patients expecting antibiotics & $54.91 \pm 22.59$ & $50.23 \pm 21.48$ & $56.20 \pm 22.75$ & 0.001 \\
\hline Degree of impacts of patient expectation on antibiotic prescribing & $43.34 \pm 26.41$ & $40.74 \pm 25.48$ & $44.05 \pm 26.64$ & 0.204 \\
\hline Total score of perceived patient pressure for antibiotics & $25.63 \pm 21.41$ & $22.22 \pm 21.40$ & $26.57 \pm 21.47$ & 0.026 \\
\hline \multicolumn{5}{|l|}{ Time pressure (Mean \pm SD) } \\
\hline Length of consultant per visit (Minutes) & $10.58 \pm 6.47$ & $12.00 \pm 6.91$ & $10.18 \pm 6.30$ & 0.007 \\
\hline
\end{tabular}

*Chi-square (fisher exact) tests for binary variables, Wilcoxon rank-sum tests for continuous variable without normal distribution and t tests for continuous variable with normal distribution; Boldface figures indicate the significant differences between physicians in UCHCS and those in RTHs

also associated with individual characteristics (gender, education and income) of physicians and institutional environments (location and socioeconomic status).

\section{Strengths and weaknesses of the study}

This study tested both intrinsic and external determinants of antibiotic prescribing through a two-level path analysis based on the TAPBM. Such an approach allowed us to examine antibiotic prescribing practices in a comprehensive and systematic way, tapping into factors associated with prescribers, patients, and institutional environments [8]. We were able to link prescribing data with survey data on prescribers, which has commonly been absent in 


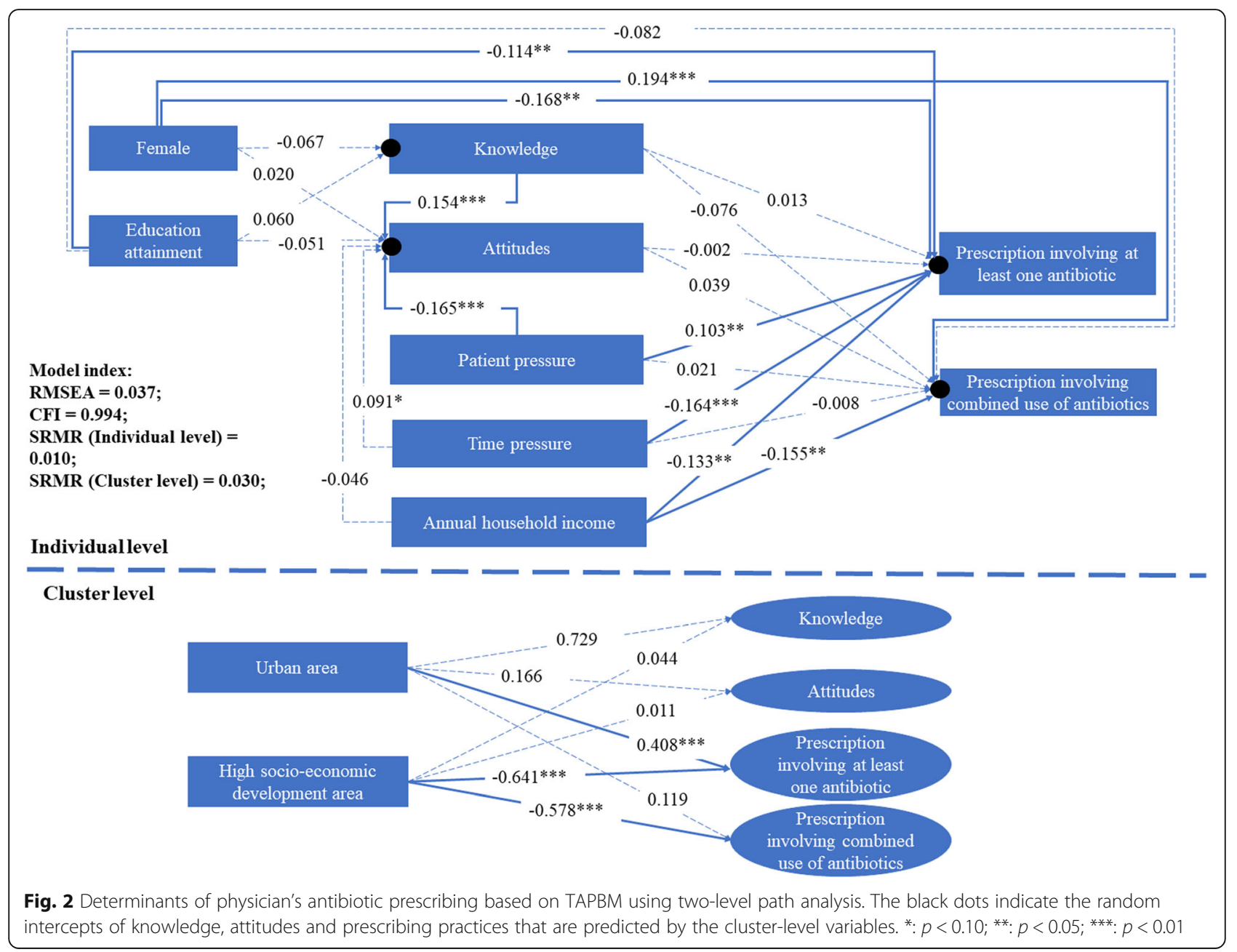

previous studies [10-24]. We also adopted well-validated instruments for measuring knowledge and attitudes of physicians, addressing problems embedded in some previous studies [43].

There are several limitations in this study. Firstly, this study was conducted in Hubei, a middle-high income region. Generalization of the findings of this study to other regions should be cautious. Secondly, in this study, prescribing indicators were not risk-adjusted due to unavailability of data regarding patient conditions. Thirdly, there is a consensus that the pharmaceutical industry may play an important role in driving prescribing decisions of physicians. The Chinese government has attempted to delink financial incentives associated with prescribing practices. However, measuring such links is difficult, if not impossible. We could only use household income as a proxy indicator in this study. Finally, there is large standard deviation of some parameters in current study, indicating a huge difference in physician's knowledge, attitudes and practices. The situation may imply that several latent classes of physicians, and among which a specific group of physicians with poorer knowledge and attitudes may play an important role contributing to the high irrational prescribing of antibiotics. However, the analysis is out of reach of the current study and further studies are warranted.

\section{Comparison to other studies \\ Effects of intrinsic factors}

Knowledge and attitudes may play different roles in prescribing practices under different system contexts. This study found no direct links between "knowledge and attitudes" and antibiotic prescribing practices, similar to the findings of a study of general practitioners (GPs) in Scotland [16]. But in Spain, appropriate use of antibiotics in clinical practices was found to be closed associated with the specific knowledge and attitudes of primary care physicians [21].

Clearly, interventions targeting prescribers alone on their knowledge and attitudes are not enough to curb over-prescriptions of antibiotics. In another study using the theory of planned behavior, we also found that 
antibiotic prescribing is not under the volitional control of physicians [39].

Individual characteristics of prescribers can shape antibiotic prescribing practices. Several studies revealed that female physicians are less likely to prescribe antibiotics $[44,45]$, consistent with the findings of this study. But female prescribers are more likely to combine antibiotics when they prescribe antibiotics. Roter and colleagues concluded in a systematic review that such a prescribing style is likely to be associated with the more patient-centered communication style of female physicians [46]. Female prescribers found it easier to implement the "waitand-see" policy on antibiotic prescribing but harder to refuse patient requests compared with their male counterparts [45].

Higher medical qualifications were found to be associated with less antibiotic prescriptions in this study after adjustments of knowledge levels. This may be attributable to the professional culture. Formal and systematic medical training may boost the confidence of practitioners [47] and encourage physicians to consider a greater variety of interventional strategies [48].

\section{Effects of external factors}

It appears that external factors play a more important role in predicting antibiotic prescribing. Similar to findings of other studies [25, 49-52], this study revealed that patient pressure is a major driver of antibiotic prescriptions. In China, defensive clinical practices are common [53]. The poor health literacy of consumers and a lack of trust in medical service providers is often blamed for fueling over-prescriptions of antibiotics.

There is no direct evidence in this study to prove that antibiotic prescribing practices are associated with financial incentives. However, low household income of physicians was found to be associated with high antibiotic prescriptions. Such a link was also documented in some previous studies [54]. Despite a significant increase in governmental investment on primary care in China, two-thirds of the budget of primary care facilities still needs to be recovered by service revenues [54]. This may motivate primary care physicians to seek extra income through over-provision of services.

The institutional variations in antibiotic prescribing practices are concerning. Rural health facilities and those servicing communities with a low socioeconomic status are relatively poorly resourced. But they are more likely to prescribe antibiotics than their better-off counterparts. China is not alone. Similar phenomena were also observed in Italy [34], Switzerland [55] and Germany [56]. This has significant implications on the effective use of the already scant health resources in the worse-off communities [57].

\section{Policy implications}

Policy interventions targeting external factors associated with antibiotic prescribing are essential. Although medical education by itself is important [58], it can only be adopted as a long term strategy. The current focus and investment on continuing education [59] and development of clinical guidelines [60] will have limited effects if external determinants of antibiotic prescribing are not properly addressed $[7,35,61,62]$.

To address the external determinants revealed in this study, several interventional strategies should be highlighted according to the empirical evidence published in the literature:

(1) Better communication skills and "wait-and-see" approach may help physicians to deal with patient pressure for antibiotics [63-65]. A study in Guangxi of China confirmed the value of communication training programs in reducing antibiotic prescriptions in pediatric care [66].

(2) Patients can be educated through social marketing campaigns to improve their understanding on the use of antibiotics and subsequently reduce requests for antibiotics [67]. However, it is challenging to change the public stereotype of antibiotics as panacea. Some pilot trials in patient education using leaflets [66] or public reporting [68] have demonstrated limited efforts in China.

(3) There is a need to improve governance and management competencies in primary care. The funding system should encourage prescribers to make decisions based on the clinical conditions of their patients, not financial gains [69, 70]. A professional culture needs to be nurtured. Empirical evidence shows that antibiotic prescribing decisions are often influenced by peers. Antibiotic prescribing can be significantly reduced when a prescriber perceives her/himself as an outlier compared with colleagues [71].

\section{Conclusion}

Antibiotic prescribing is a complex process shaped by both intrinsic (knowledge, attitudes and individual characteristics) and external (patients, institutions and healthcare systems) factors. A systems approach is required to curb over-prescription of antibiotics in China. Apart from educating prescribers, it is equally important, if not more, to educate patients to ease the pressure from patient requests. Management also plays an important role in changing prescribing behaviors.

The link between high use of antibiotics and low socioeconomic status is concerning. Further studies are warranted to explore the underlying reasons of higher antibiotic use in rural and low socioeconomic settings in China. 


\section{Additional file}

Additional file 1: Table S1. Survey instrument. (DOCX 33 kb)

\begin{abstract}
Abbreviations
AR: Antibiotic resistance; CFI: comparative fit index; GDP: Gross domestic product; ICC: Intra-cluster correlation coefficient; KAAR-11: 11-item Knowledge and Attitudes regarding Antibiotics and Resistance; KAP: Knowledge-attitudes-practices; MLR: Maximum likelihood with robust standard error; RMSEA: Root mean square error of approximation; RTHCs: Rural township health centers; SD: Standard deviation; SEM: Structural equation modelling;; SRMR: Standardized root mean squared residual; TAPBM: Teixeira Antibiotic Prescribing Behavioral Model; UCHCs: Urban community health centers; WHO: World Health Organization
\end{abstract}

\section{Acknowledgements}

The authors would like to sincerely thank the participating physicians and the support from managers of the included institutions and local governments.

\section{Authors' contributions}

$X Z$ designed the project and participated in the collection and interpretation of data. CL contributed to the acquisition, analysis and interpretation of data and drafted the manuscript. CL participated in data analysis, interpretation of results, and writing of the manuscript. DW participated in the cleaning and interpretation of data. All authors have read and approved the final version of the article.

\section{Funding}

This study was funded by the National Natural Science Foundation of China (grant no. 71373092).

The funding body played no part in the study design, collection, analysis and interpretation of data, writing of the manuscript or the decision to submit the manuscript for publication.

\section{Availability of data and materials}

The data that support the findings of this study are available from surveyed local institutions and governments but restrictions apply to the availability of these data, which were used under license for the current study, and so are not publicly available. Data are however available from the authors upon reasonable request and with permission of surveyed local institutions and governments.

\section{Ethics approval and consent to participate}

This study was approved by the Ethics Committee of Tongji Medical College, Huazhong University of Science and Technology (NO: IORG 0003571).

Written consent was obtained from each participant in current study.

\section{Consent for publication}

Written consent for publication was obtained from each participant prior to the survey.

\section{Competing interests}

The authors declare no conflict of interest.

\section{Author details}

${ }^{1}$ School of Medicine and Health Management, Tongji Medical School, Huazhong University of Science and Technology, Wuhan, Hubei, China. ${ }^{2}$ School of Psychology and Public Health, La Trobe University, Melbourne, Victoria, Australia.

Received: 18 June 2019 Accepted: 31 July 2019

Published online: 07 August 2019

\section{References}

1. WHO. Global action plan on antimicrobial resistance. Geneva, Switzerland: World Health Organization; 2015.

2. Jim ON. Review on antimicrobial resistance antimicrobial resistance: tackling a crisis for the health and wealth of nations. London: Review on Antimicrobial Resistance; 2014.
3. Goossens H, Ferech M, Vander Stichele R, Elseviers M. Outpatient antibiotic use in Europe and association with resistance: a cross-national database study. Lancet. 2005;365(9459):579-87.

4. Costelloe C, Metcalfe C, Lovering A, Mant D, Hay AD. Effect of antibiotic prescribing in primary care on antimicrobial resistance in individual patients: systematic review and meta-analysis. Bmj. 2010;340:c2096.

5. Shallcross LJ, Davies DS. Antibiotic overuse: a key driver of antimicrobial resistance. The British journal of general practice : the journal of the Royal College of General Practitioners. 2014;64(629):604-5.

6. Shapiro DJ, Hicks LA, Pavia AT, Hersh AL. Antibiotic prescribing for adults in ambulatory care in the USA, 2007-09. J Antimicrob Chemother. 2014;69(1): 234-40.

7. Wang J, Wang P, Wang X, Zheng Y, Xiao Y. Use and prescription of antibiotics in primary health care settings in China. JAMA Intern Med. 2014; 174(12):1914-20.

8. Teixeira Rodrigues A, Roque F, Falcão A, Figueiras A, Herdeiro MT. Understanding physician antibiotic prescribing behaviour: a systematic review of qualitative studies. Int J Antimicrob Agents. 2013;41(3):203-12.

9. Lopez-Vazquez P, Vazquez-Lago JM, Figueiras A. Misprescription of antibiotics in primary care: a critical systematic review of its determinants. J Eval Clin Pract. 2012;18(2):473-84.

10. Grossman Z, del Torso S, Hadjipanayis A, van Esso D, Drabik A, Sharland M. Antibiotic prescribing for upper respiratory infections: European primary paediatricians' knowledge, attitudes and practice. Acta Paediatr. 2012;101(9): 935-40.

11. Om C, Vlieghe E, McLaughlin JC, Daily F, McLaws ML. Antibiotic prescribing practices: a national survey of Cambodian physicians. Am J Infect Control. 2016:44(10):1144-8.

12. García C, Llamocca LP, García K, Jiménez A, Samalvides F, Gotuzzo E, Jacobs J. Knowledge, attitudes and practice survey about antimicrobial resistance and prescribing among physicians in a hospital setting in Lima, Peru. BMC Clin Pharmacol. 2011;11(1):18.

13. Quet F, Vlieghe E, Leyer C, Buisson Y, Newton PN, Naphayvong P, Keoluangkhot V, Chomarat M, Longuet C, Steenkeste N, et al. Antibiotic prescription behaviours in Lao People's Democratic Republic: a knowledge, attitude and practice survey. Bull World Health Organ. 2015;93(4):219-27.

14. Pulcini C, Williams F, Molinari N, Davey P, Nathwani D. Junior doctors' knowledge and perceptions of antibiotic resistance and prescribing: a survey in France and Scotland. Clinical microbiology and infection : the official publication of the European Society of Clinical Microbiology and Infectious Diseases. 2011;17(1):80-7.

15. Hassali MA, Kamil TK, Md Yusof FA, Alrasheedy AA, Yusoff ZM, Saleem F, AlTamimi SK, Wong ZY, Aljadhey H, Godman B. General practitioners' knowledge, attitude and prescribing of antibiotics for upper respiratory tract infections in Selangor, Malaysia: findings and implications. Expert Rev AntiInfect Ther. 2015;13(4):511-20.

16. Eccles MP, Grimshaw JM, Johnston M, Steen N, Pitts NB, Thomas R, Glidewell E, Maclennan G, Bonetti D, Walker A. Applying psychological theories to evidence-based clinical practice: identifying factors predictive of managing upper respiratory tract infections without antibiotics. Implement Sci. 2007;2(1):26

17. McCullough AR, Rathbone J, Parekh S, Hoffmann TC, Del Mar CB. Not in my backyard: a systematic review of clinicians' knowledge and beliefs about antibiotic resistance. J Antimicrob Chemother. 2015;70(9):2465-73.

18. Thakolkaran N, Shetty AV, D'Souza NDR, Shetty AK. Antibiotic prescribing knowledge, attitudes, and practice among physicians in teaching hospitals in South India. Journal of family medicine and primary care. 2017;6(3):526-32.

19. Tan WL, Siti R, Shahfini I, Zuraidah A. Knowledge, attitude and practice of antibiotics prescribing among medical officers of public health care facilities in the state of Kedah, Malaysia. Med J Malaysia. 2015;70(5):307-11.

20. Sahin $H$, Arsu G, Köseli D, Büke $C$. evaluation of primary health care physicians' knowledge on rational antibiotic use. Mikrobiyoloji bulteni. 2008; 42(2):343-8.

21. Gonzalez-Gonzalez C, López-Vázquez P, Vázquez-Lago JM, Piñeiro-Lamas M, Herdeiro MT, Arzamendi PC, Figueiras A, Group G. Effect of Physicians' attitudes and knowledge on the quality of antibiotic prescription: a cohort study. PLoS One. 2015;10(10):e0141820.

22. Thriemer $K$, Katuala $Y$, Batoko B, Alworonga JP, Devlieger H, Van Geet C, Ngbonda D, Jacobs J. Antibiotic prescribing in DR Congo: a knowledge, attitude and practice survey among medical doctors and students. PLoS One. 2013;8(2):e55495. 
23. Shehadeh M, Suaifan G, Darwish RM, Wazaify M, Zaru L, Alja'fari S. Knowledge, attitudes and behavior regarding antibiotics use and misuse among adults in the community of Jordan. A pilot study. Saudi Pharm J. 2012;20(2):125-33.

24. Cho H-J, Hong S-J, Park S. Knowledge and beliefs of primary care physicians, pharmacists, and parents on antibiotic use for the pediatric common cold. Soc Sci Med. 2004;58(3):623-9.

25. McKay R, Mah A, Law MR, McGrail K, Patrick DM. Systematic review of factors associated with antibiotic prescribing for respiratory tract infections. Antimicrob Agents Chemother. 2016;60(7):4106-18.

26. National Bureau of Statistics of China. National data. In. http://data.stats.gov. cn; National Bureau of Statistics of China; 2018.

27. Liu C, Zhang X, Wang X, Zhang X, Wan J, Zhong F: Does public reporting influence antibiotic and injection prescribing to all patients? A clusterrandomized matched-pair trial in China. Medicine 2016, 95(26):e3965-e3965.

28. Sirota M, Round T, Samaranayaka S, Kostopoulou O. Expectations for antibiotics increase their prescribing: causal evidence about localized impact. Health psychology : official journal of the Division of Health Psychology, American Psychological Association. 2017;36(4):402-9.

29. Cole A. GPs feel pressurised to prescribe unnecessary antibiotics, survey finds. BMJ : British Medical Journal. 2014;349:g5238.

30. Yang L, Liu C, Ferrier JA, Zhang X. Organizational barriers associated with the implementation of national essential medicines policy: a cross-sectional study of township hospitals in China. Soc Sci Med. 2015;145:201-8.

31. Gulliford MC, Prevost AT, Charlton J, Juszczyk D, Soames J, McDermott L, Sultana K, Wright M, Fox R, Hay AD, et al. Effectiveness and safety of electronically delivered prescribing feedback and decision support on antibiotic use for respiratory illness in primary care: REDUCE cluster randomised trial. BMJ. 2019;364:1236.

32. Broom A, Broom J, Kirby E. Cultures of resistance? A Bourdieusian analysis of doctors' antibiotic prescribing. Soc Sci Med. 2014;110:81-8.

33. Touboul-Lundgren P, Jensen S, Drai J, Lindbæk M: Identification of cultural determinants of antibiotic use cited in primary care in Europe: a mixed research synthesis study of integrated design "culture is all around us". BMC Public Health 2015, 15:908-908.

34. Russo V, Monetti VM, Guerriero F, Trama U, Guida A, Menditto E, Orlando $\checkmark$. Prevalence of antibiotic prescription in southern Italian outpatients: real-world data analysis of socioeconomic and sociodemographic variables at a municipality level. ClinicoEconomics and Outcomes Research. 2018;10:251-8.

35. Yin X, Song F, Gong Y, Tu X, Wang Y, Cao S, Liu J, Lu Z. A systematic review of antibiotic utilization in China. J Antimicrob Chemother. 2013; 68(11):2445-52

36. WHO. Promoting rational use of medicines: core components. In: Geneva: World Health Organization; 2002.

37. Garcia C, Llamocca LP, Garcia K, Jimenez A, Samalvides F, Gotuzzo E, Jacobs J. Knowledge, attitudes and practice survey about antimicrobial resistance and prescribing among physicians in a hospital setting in Lima, Peru. BMC clinical pharmacology. 2011;11:18.

38. Lopez-Vazquez P, Vazquez-Lago JM, Gonzalez-Gonzalez C, Pineiro-Lamas M, Lopez-Duran A, Herdeiro MT, Figueiras A. Development and validation of the knowledge and attitudes regarding antibiotics and resistance (KAAR-11) questionnaire for primary care physicians. J Antimicrob Chemother. 2016; 71(10):2972-9.

39. Liu C, Liu C, Wang D, Deng Z, Tang Y, Zhang X: Determinants of antibiotic prescribing behaviors of primary care physicians in Hubei of China: a structural equation model based on the theory of planned behavior. Antimicrob Resist Infect Control 2019, 8:23-23.

40. Hu LT, Bentler PM. Cutoff criteria for fit indexes in covariance structure analysis: conventional criteria versus new alternatives. Struct Equ Model. 1999;6(1):1-55.

41. Bentler PM. Comparative fit indexes in structural models. Psychol Bull. 1990; 107(2):238-46.

42. WHO. Using indicators to measure country pharmaceutical situations: fact book on WHO level one and level two monitoring indicators. Geneva: World Health Organization; 2006.

43. Alumran A, Hou X-Y, Hurst C. Validity and reliability of instruments designed to measure factors influencing the overuse of antibiotics. Journal of Infection and Public Health. 2012;5(3):221-32.

44. Chamany S, Schulkin J, Rose CE Jr, Riley LE, Besser RE. Knowledge, attitudes, and reported practices among obstetrician-gynecologists in the USA regarding antibiotic prescribing for upper respiratory tract infections. Infect Dis Obstet Gynecol. 2005;13(1):17-24.

45. Eggermont D, Smit MAM, Kwestroo GA, Verheij RA, Hek K, Kunst AE. The influence of gender concordance between general practitioner and patient on antibiotic prescribing for sore throat symptoms: a retrospective study. BMC Fam Pract. 2018;19(1):175.

46. Roter DL, Hall JA, Aoki Y. Physician gender effects in medical CommunicationA meta-analytic review. JAMA. 2002;288(6):756-64.

47. Pulcini C, Gyssens IC. How to educate prescribers in antimicrobial stewardship practices. Virulence. 2013;4(2):192-202.

48. Song $P$, Jin C, Tang W. New medical education reform in China: towards healthy China 2030. Bioscience trends. 2017;11(4):366-9.

49. Dempsey PP, Businger AC, Whaley LE, Gagne JJ, Linder JA. Primary care clinicians' perceptions about antibiotic prescribing for acute bronchitis: a qualitative study. BMC Fam Pract. 2014;15(1):194.

50. Fletcher-Lartey S, Yee M, Gaarslev C, Khan R. Why do general practitioners prescribe antibiotics for upper respiratory tract infections to meet patient expectations: a mixed methods study. BMJ Open. 2016; 6(10):e012244.

51. Lum EPM, Page K, Whitty JA, Doust J, Graves N. Antibiotic prescribing in primary healthcare: dominant factors and trade-offs in decision-making. Infection, Disease \& Health. 2018;23(2):74-86.

52. Mustafa M, Wood F, Butler CC, Elwyn G. Managing expectations of antibiotics for upper respiratory tract infections: a qualitative study. Ann Fam Med. 2014;12(1):29-36.

53. He AJ. The doctor-patient relationship, defensive medicine and overprescription in Chinese public hospitals: evidence from a cross-sectional survey in Shenzhen city. Soc Sci Med. 2014;123:64-71.

54. Wang J, Wang P, Wang X, Zheng Y, Xiao Y. Use and prescription of antibiotics in primary health care settings in ChinaAntibiotics use in primary health care in ChinaAntibiotics use in primary health Care in China. JAMA Intern Med. 2014;174(12):1914-20.

55. Filippini M, Masiero G, Moschetti K. Socioeconomic determinants of regional differences in outpatient antibiotic consumption: evidence from Switzerland. Health policy (Amsterdam, Netherlands). 2006;78(1): 77-92.

56. Kern W, de With K, Nink K, Steib-Bauert M, Schroder H. Regional variation in outpatient antibiotic prescribing in Germany. Infection. 2006;34(5):269-73.

57. Sianesi B, Reenen JV. The returns to education: macroeconomics. J Econ Surv. 2003;17(2):157-200.

58. Kramer MHH, Tichelaar J, Caris MG, van Agtmael MA, Schutte T, Sikkens JJ. Improving antibiotic prescribing skills in medical students: the effect of elearning after 6 months. J Antimicrob Chemother. 2018;73(8):2243-6.

59. Ministry of Health. Administrative Measures for the Clinical Use of Antibacterial Drugs. In. Edited by Health Mo. Beijing: Ministry of Health; 2012.

60. Nanshan Z, Xirun W, Xiaojun M, Chen W, Rui W, Dayou W, al. E: Guiding principles for clinical application of antimicrobial agents (2015 version) In. Edited by China NHaFPCPsRo. Beijing; 2015.

61. Li Y, Xu J, Wang F, Wang B, Liu L, Hou W, Fan H, Tong Y, Zhang J, Lu Z. Overprescribing in China, driven by financial incentives, results in very high use of antibiotics, injections, and corticosteroids. Health affairs (Project Hope). 2012;31(5):1075-82.

62. Mao W, Vu H, Xie Z, Chen W, Tang S. Systematic review on irrational use of medicines in China and Vietnam. PLoS One. 2015;10(3):e0117710.

63. van der Velden AW, Pijpers E, Kuyvenhoven MM, Tonkin-Crine SK, Little P, Verheij TJ. Effectiveness of physician-targeted interventions to improve antibiotic use for respiratory tract infections. Br J Gen Pract. 2012;62(605):e801-7.

64. Arnold SR, Straus SE. Interventions to improve antibiotic prescribing practices in ambulatory care. The Cochrane database of systematic reviews. 2005;4 Cd003539

65. Cross EL, Tolfree R, Kipping R. Systematic review of public-targeted communication interventions to improve antibiotic use. J Antimicrob Chemother. 2017;72(4):975-87.

66. Wei X, Zhang Z, Walley JD, Hicks JP, Zeng J, Deng S, Zhou Y, Yin J, Newell JN, Sun $\mathrm{Q}$, et al. Effect of a training and educational intervention for physicians and caregivers on antibiotic prescribing for upper respiratory tract infections in children at primary care facilities in rural China: a cluster-randomised controlled trial. Lancet Glob Health. 2017;5(12):e1258-67.

67. Edgar T, Boyd SD, Palame MJ. Sustainability for behaviour change in the fight against antibiotic resistance: a social marketing framework. J Antimicrob Chemother. 2009;63(2):230-7. 
68. Liu C, Zhang X, Wan J. Public reporting influences antibiotic and injection prescription in primary care: a segmented regression analysis. J Eval Clin Pract. 2015;21(4):597-603

69. Yi H, Miller G, Zhang L, Li S, Rozelle S. Intended and unintended consequences of China's zero markup drug policy. Health affairs (Project Hope). 2015;34(8):1391-8.

70. He Y, Dou G, Huang Q, Zhang X, Ye Y, Qian M, Ying X: Does the leading pharmaceutical reform in China really solve the issue of overly expensive healthcare services? Evidence from an empirical study. PLoS One 2018, 13(1):e0190320-e0190320.

71. Hallsworth M, Chadborn T, Sallis A, Sanders M, Berry D, Greaves F, Clements $L$, Davies SC. Provision of social norm feedback to high prescribers of antibiotics in general practice: a pragmatic national randomised controlled trial. Lancet (London, England). 2016;387(10029):1743-52.

\section{Publisher's Note}

Springer Nature remains neutral with regard to jurisdictional claims in published maps and institutional affiliations.

Ready to submit your research? Choose BMC and benefit from:

- fast, convenient online submission

- thorough peer review by experienced researchers in your field

- rapid publication on acceptance

- support for research data, including large and complex data types

- gold Open Access which fosters wider collaboration and increased citations

- maximum visibility for your research: over $100 \mathrm{M}$ website views per year

At BMC, research is always in progress.

Learn more biomedcentral.com/submissions 